\title{
The structure of diaminodurene and the dynamics of the methyl groups
}

L. Sobczyk, M. Prager, W. Sawka-Dobrowolska, G. Bator, A. Pawlukojć, E. Grech, L. van Eijck, A. Ivanov, S. Rols, J. Wuttke, and T. Unruh

Citation: The Journal of Chemical Physics 130, 164519 (2009); doi: 10.1063/1.3125629

View online: https://doi.org/10.1063/1.3125629

View Table of Contents: http://aip.scitation.org/toc/jcp/130/16

Published by the American Institute of Physics

\section{Articles you may be interested in}

Hydration-dependent dynamics of human telomeric oligonucleotides in the picosecond timescale: A neutron scattering study

The Journal of Chemical Physics 143, 015102 (2015); 10.1063/1.4923213

Neutron scattering study of the dynamics of a polymer melt under nanoscopic confinement

The Journal of Chemical Physics 131, 174901 (2009); 10.1063/1.3258329

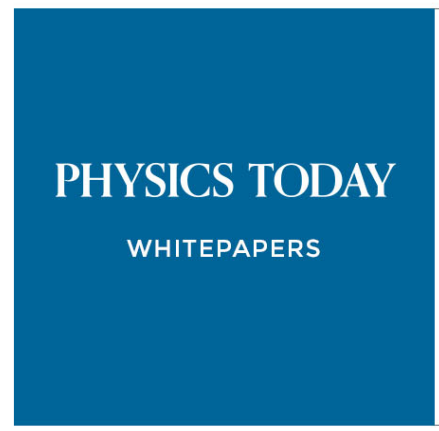




\title{
The structure of diaminodurene and the dynamics of the methyl groups
}

\author{
L. Sobczyk, ${ }^{1}$ M. Prager, ${ }^{2}$ W. Sawka-Dobrowolska, ${ }^{1}$ G. Bator ${ }^{1, a)}$ A. Pawlukojć, ${ }^{3}$ E. Grech, ${ }^{4}$ \\ L. van Eijck, ${ }^{5}$ A. Ivanov, ${ }^{5}$ S. Rols, ${ }^{5}$ J. Wuttke, ${ }^{6}$ and T. Unruh ${ }^{7}$ \\ ${ }^{1}$ Faculty of Chemistry, University of Wroctaw, 50383 Wroctaw, Poland \\ ${ }^{2}$ Institut für Festkörperforschung, Forschungszentrum Jülich GmbH, 52425 Jülich, Germany \\ ${ }^{3}$ Institute of Nuclear Chemistry and Technology, 03-195 Warsaw, Poland \\ ${ }^{4}$ West Pomeranian University of Technology, 70-310 Szczecin, Poland \\ ${ }^{5}$ Institut Laue Langevin, 38042 Grenoble, France \\ ${ }^{6}$ Institut für Festkörperforschung, Forschungszentrum Jülich GmbH, Jülich Centre for Neutron Science \\ at FRM II, Lichtenbergstr. 1, 85747 Garching, Germany \\ ${ }^{7}$ Forschungsneutronenquelle Heinz Maier-Leibnitz, (FRMII), Technische Universität München, \\ Lichtenbergstr. 1, 85747 Garching, Germany
}

(Received 24 November 2008; accepted 4 April 2009; published online 30 April 2009)

\begin{abstract}
Diaminodurene crystallizes in the orthorhombic space group $P b c a$, with eight molecules in the unit cell. Four inequivalent methyl groups with different environments exist in a molecule. The amino groups are also different, which is well reflected in infrared spectra. Two tunneling modes are resolved at 23.7 and $7.0 \mu \mathrm{eV}$ at $4.5 \mathrm{~K}$. Their intensities are consistent with the presence of two further unresolved tunneling modes. Quasielastic spectra are composed of three Lorentzians of equal intensities. The two low activation energies and tunnel modes are modeled into consistent rotational potentials. The third activation energy and a librational band are used to guess the strength of the two stronger rotational potentials. The internal modes related to the torsional/librational vibrations mix with ring torsions in the range of $70-220 \mathrm{~cm}^{-1}$. This way the tunnel modes couple to ring torsions whose energy determines the broadening of both tunnel bands. The calculations for free molecules yield mode frequencies a little bit lower than the experimental inelastic neutron scattering (INS) values. Application of theoretical methods elaborated for the crystalline state leads to a satisfactory consistency. It is also valid for bending modes of $\mathrm{NH}_{2}$ groups, which in the solid state show much higher frequencies than in the gas phase, as expected. (C) 2009 American Institute of Physics. [DOI: 10.1063/1.3125629]
\end{abstract}

\section{INTRODUCTION}

Inelastic neutron scattering techniques can be applied to the study of the dynamics of methyl groups in various compounds. Using thermal neutrons a determination of the vibrational density of states is possible in the regime of internal torsional/librational vibrations located at low frequencies and characterized by high intensities expressed in the scattering function $S(Q, \omega) .^{1-3}$ With cold neutrons on the other hand quasi-elastic neutron scattering (QENS) spectra are obtained describing stochastic, classical reorientational jumps over the barrier. The activation energy represents a further important information on the rotational potential. ${ }^{4,5}$ If, finally, the rotational barrier is not very high, the tunneling splitting located energetically in the $\mu \mathrm{eV}$ region is a third and the most sensitive eigenenergy in estimating the barrier. ${ }^{6,7}$

The correlations between the inelastic neutron scattering (INS) experimental data and the rotational potential were analyzed in Refs. 8 and 9. These techniques were recently applied in studies on methyl derivatives of benzene and pyrazine and their charge transfer (CT) and hydrogen bonded complexes. ${ }^{10-15}$ The aim of these investigations was a search for factors affecting the rotational potential in real systems. The lattice effect through the direct interaction with neigh-

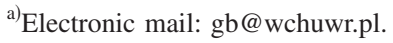

boring atoms is one of the main factors. Thus the complexation should affect the behavior of methyl groups that has been shown in the cited papers. The other factors, as resulted from the theoretical analysis, ${ }^{13}$ should be the CT from the methyl derivative, as a donor, to the complex component as an electron acceptor or a proton donor.

Diaminodurene (DAD) is a particularly interesting compound from the family of the durene and pyrazine derivatives. According to the redox potential, ${ }^{16}$ it is a strong electron donor. A comparison of the redox properties with the other compounds of this type was performed. The oxidation of DAD leads to the formation of Wurster's salts-free radicals characterized by the absorption band located below $\lambda$ $=500 \mu \mathrm{m} .{ }^{17}$ The free radical state is well reflected in electron spin resonance (ESR) spectra. ${ }^{18}$ As a compound easily oxidizing, DAD was used in construction of photomicrobial biofuel cells as a mediator in the anode compartment. ${ }^{19}$ One should mention that DAD was used in studies of programmed cell death processes. ${ }^{20}$

The aim of the present work was to study the tunneling splitting, QENS, and the vibrational density of state of DAD. Because the structure of DAD was not known we decided first to determine the x-ray crystal structure and then to analyze the packing in the lattice and the structural parameters of a molecule. The aim of the work was also to analyze the IR spectra based on the calculations of frequencies and in- 
tensities of internal modes. This information was also important for the description of methyl group torsional modes in the analysis of INS spectra.

\section{THEORY}

The standard description of rotational tunneling and quasielastic scattering is the single particle model (SPM). ${ }^{7}$ SPM represents a mean field theory. The environment of the molecule is represented by a rotational potential $V(\varphi)$. Due to the symmetry of the methyl group it is expressed in form of a Fourier expansion of threefold symmetry up to order of $J$

$$
V(\varphi)=\sum_{j=1}^{J} \frac{V_{3 j}}{2}\left(1-\cos \left(3 j \varphi-\varphi_{3 j}\right)\right) .
$$

Usually the expansion is limited to $J=2$. At low temperatures, when the classical rotational jumps are frozen, this potential determines the excitations of the hindered quantum rotor by the eigenvalues $E_{i}$ of the stationary single particle Schrödinger equation ${ }^{6,7}$

$$
\left\{-B \frac{\partial^{2}}{\partial \varphi^{2}}+V(\varphi)\right\} \Psi_{i}=E_{i} \Psi_{i} .
$$

Here $B=\hbar^{2} / 2 I=655 \mu \mathrm{eV}$ is the rotational constant of the methyl group with the moment of inertia $I$. The tunnel splitting is the difference between the two lowest levels, $\hbar \omega_{t}$ $=E_{1}-E_{o}$. If further transitions to higher rotational states $\left(E_{2}, E_{3}, \ldots\right)$ are known, one can refine the rotational potential by introducing higher Fourier terms.

High resolution neutron spectroscopy is an especially successful technique to observe the low energy transitions of weakly hindered rotors. The groundstate tunnel splitting $\hbar \omega_{t}$ gives rise to a simple scattering function. ${ }^{6,7}$ In the present example of DAD we have four inequivalent methyl groups. The scattering from the rest of the molecule is dominated by $n_{p}=4$ individual protons in the amino groups. These protons scatter only elastically. Thus we obtain the scattering function

$$
\begin{aligned}
S(Q, \omega)= & \left(4\left(\frac{5}{3}+\frac{4}{3} j_{o}(Q d)\right)+n_{p}\right) \delta(\omega) \\
& +\sum_{n=1}^{4}\left(\frac{2}{3}-\frac{2}{3} j_{o}(Q d)\right)\left\{\delta\left(\omega+\omega_{t n}\right)+\delta\left(\omega-\omega_{t n}\right)\right\}
\end{aligned}
$$

with momentum transfer $Q$ and distance $d$ of the protons in the methyl group. For fully resolved tunneling bands the theory yields a ratio $\Sigma I_{\text {inel }} / I_{\mathrm{el}}=8-8 j_{o} / 20+16 j_{o}+3 n_{p}$. This quantity shall be compared to the experiment below. If a tunnel splitting is not resolved the related inelastic transition is included in the elastic line with an obvious modification of Eq. (3).

At higher temperatures, thermal fluctuations of the lattice become significant, and it becomes appropriate to model the rotational dynamics with classical jumps. Each jump process is described by a Lorentzian. With four different methyl groups the scattering function is

$$
\begin{aligned}
S(Q, \omega)= & \left(4\left(1+2 j_{o}(Q d)\right)+n_{p}\right) \delta(\omega) \\
& +\sum_{n=1}^{4}\left(2-2 j_{o}(Q d)\right) L\left(\omega, \Gamma_{n}\right) .
\end{aligned}
$$

The linewidths $\Gamma_{n}$ of the Lorentzians $L\left(\omega, \Gamma_{n}\right)$ follow Arrhenius dependencies with activation energies $E_{a n}$ representing the distance from the rotational groundstate to the maximum of the potential $V_{n}(\varphi)$,

$$
\Gamma_{n}=\Gamma_{o n} \exp \left(\frac{E_{a n}}{k T}\right) .
$$

\section{EXPERIMENTAL AND CALCULATION}

\section{A. Sample preparation}

The sample of DAD was bought from Fluka and used for all experiments without additional treatment.

\section{B. Crystallography}

The $\mathrm{X}$-ray diffraction studies were performed on a Kuma KM4CCD $\kappa$-axis four circle diffractometer equipped with Oxford Cryosystem Cooler using graphite monochromated Mo $K \alpha$ radiation. The data were corrected for Lorentz and polarization effects.

The structure was solved by direct methods with SHELXS-97 (Ref. 21) and refined by the full-matrix leastsquares methods using the SHELXL-97 (Ref. 22) program. Nonhydrogen atoms were refined with anisotropic thermal parameters. All the $\mathrm{H}$ atoms were found from difference synthesis and refined with isotropic thermal parameters. The crystal data and structure refinements are summarized in Table I.

\section{Infrared spectra}

The infrared spectra were recorded in Nujol or Fluorolube suspensions (KBr or CsI windows) using a Fourier transform infrared (FTIR) Bruker IFS 113v spectrophotometer with a resolution of $2 \mathrm{~cm}^{-1}$.

\section{Raman spectra}

The Raman spectra of powder samples were recorded on a Nicolet Magma 960 FT Raman spectrometer. A diodepumped Nd:YAG laser was used as an excitation source with a power of approximately $200 \mathrm{~mW}$. Backscattering geometry was applied. The resolution was set up for $2 \mathrm{~cm}^{-1} ; 512$ scans were measured.

\section{E. Neutron scattering studies}

High resolution spectra were measured on the backscattering spectrometers IN16 of Institut Laue-Langevin (ILL), ${ }^{23}$ Grenoble, and on SPHERES ${ }^{24,25}$ of the Jülich Centre for Neutron Science at Forschungsneutronenquelle Heinz Maier-Leibnitz (FRMII), Technische Universität München (TUM), Garching. The main part of quasielastic neutron spectra was obtained with the TOFTOF spectrometer $^{26}$ also placed at FRMII, TUM, Garching. The wavelength of $6.0 \AA$ 
TABLE I. Crystal data and structure refinement for DAD.

\begin{tabular}{|c|c|}
\hline Empirical formula & $\mathrm{C}_{10} \mathrm{H}_{16} \mathrm{~N}_{2}$ \\
\hline$F_{w}$ & 164.25 \\
\hline$T(\mathrm{~K})$ & $100(2)$ \\
\hline Wavelength $(\AA)$ & 0.71073 \\
\hline Crystal system & Orthorhombic \\
\hline Space group & Pbca \\
\hline \multicolumn{2}{|l|}{ Unit cell dimension $(\AA)$} \\
\hline$a$ & $8.579(2)$ \\
\hline$b$ & $12.719(2)$ \\
\hline$c$ & $16.819(2)$ \\
\hline$V\left(\AA^{3}\right)$ & $1835.2(6)$ \\
\hline$Z$ & 8 \\
\hline Calculated density $\left(\mathrm{g} \mathrm{cm}^{-3}\right)$ & 1.189 \\
\hline Absorption coefficient $\left(\mathrm{mm}^{-1}\right)$ & 0.072 \\
\hline$F(000)$ & 720 \\
\hline Crystal size $\left(\mathrm{mm}^{3}\right)$ & $0.40 \times 0.40 \times 0.30$ \\
\hline$\theta$ range for data collection $\left(^{\circ}\right)$ & $3.11-28.00$ \\
\hline Index ranges & $\begin{array}{l}-9 \leq h \leq 11 \\
-16 \leq k \leq 16 \\
-22 \leq l \leq 22\end{array}$ \\
\hline Ref. collected/unique & $24916 / 2211\left[R_{(\mathrm{int})}=0.039\right]$ \\
\hline Refinement method & Full-matrix least-squares on $F^{2}$ \\
\hline Data /parameters & $2211 / 173$ \\
\hline Goodness-of-fit on $F^{2}$ & 1.007 \\
\hline Final $R$ indices $[I>2 \sigma(I)]$ & $R_{1}=0.0427, w R_{2}=0.1202$ \\
\hline$R$ indices (all data) & $R_{1}=0.0577, w R_{2}=0.1252$ \\
\hline Largest diff. peak and hole $\left(e \AA^{-3}\right)$ & 0.268 and -0.164 \\
\hline
\end{tabular}

used leads to an energy resolution of $60 \mu \mathrm{eV}$. A few very high resolution QENS scans are added from IN16 measurements.

Spectra in the regime of internal modes and phonon energies-including methyl librational modes-are due to the ILL instruments ${ }^{23}$ IN1BeF (beryllium filter) and IN4 (time-of-flight), Grenoble, France. Using a set of different wavelengths, the energy regime from about 1 to $250 \mathrm{meV}$ could be explored with adapted energy resolution. On the IN1BeF, spectra were collected in the energy ranges of 14-64 meV (0.5 meV resolution), 28-128 meV (1 meV resolution), and $128-268 \mathrm{meV}$ (2 meV resolution). On IN4 were collected three incident wavelengths $(3,1.5$, and $1.0 \AA$ ). In all measurements, the lowest sample temperature was $T=10 \mathrm{~K}$.

\section{F. Calculations}

Calculations tending toward the optimized structure of an isolated DAD molecule, frequencies of normal vibrations and the intensities of corresponding bands in the infrared and Raman spectra were performed by using a GAUSSIAN 03 program $^{27}$ on the $B 3 L Y P / 6-31 G(d, p)$ level. The optimized geometry of molecules and the harmonic force field calculations for solid DAD have been performed using the DMOL3 program $^{28,29}$ as a part of MATERIALS STUDIO package. ${ }^{30}$ The results have been obtained within generalized gradient approximation (GGA) at Becke exchange ${ }^{31}$ plus Lee-YangParr correlation $^{32}$ (BLYP), PBE (Perdew-Burke-Ernzerhof correlation $^{33}$ ) and PW91 [Perdew-Wang GGA (Ref. 34)]

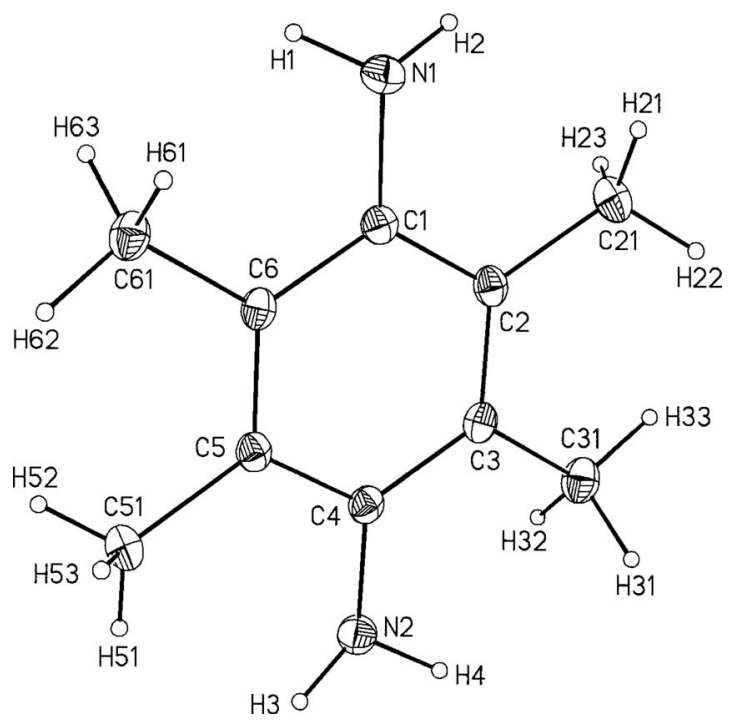

FIG. 1. The structure and atom numbering of DAD showing 35\% probability displacement ellipsoids.

functionals. Calculations have been performed using double numerical plus polarization (DNP) on nonhydrogen and hydrogen atoms) basis set as implemented in DMOL3.

\section{RESULTS AND DISCUSSION}

\section{A. X-ray diffraction}

The structure of the DAD molecule with atom numbering is presented in Fig. 1. The details connected with the $\mathrm{X}$-ray diffraction results are deposited at the Cambridge Crystallographic Data Center (CCDC No. 721211).

The core of the DAD molecule, defined by the carbon atoms of the benzene ring, is almost undistorted. This is related both to the bond lengths and angles. A marked deviation from planarity was, however, found for carbon atoms of methyl groups and nitrogen atoms of amino groups, as shown in Table II. The atoms $\mathrm{C}(21)$ and $\mathrm{C}(51)$ lie above the

TABLE II. Displacements $(\AA)$ from mean plane of benzene ring.

\begin{tabular}{lcrrr}
\hline \hline & X-ray deviation from plane & & & \\
Atom & $(\AA)$ & BLYP/DNP & PBE/DNP & PW91/DNP \\
\hline $\mathrm{C}(1)^{\mathrm{a}}$ & $-0.013(1)$ & -0.0131 & -0.0124 & -0.0126 \\
$\mathrm{C}(2)^{\mathrm{a}}$ & $0.018(1)$ & 0.0168 & 0.0179 & 0.0175 \\
$\mathrm{C}(3)^{\mathrm{a}}$ & $-0.009(1)$ & -0.0103 & -0.0123 & -0.0112 \\
$\mathrm{C}(4)^{\mathrm{a}}$ & $-0.004(1)$ & 0.0001 & 0.0010 & -0.0001 \\
$\mathrm{C}(5)^{\mathrm{a}}$ & $0.009(1)$ & 0.0037 & 0.0047 & 0.0048 \\
$\mathrm{C}(6)^{\mathrm{a}}$ & $-0.000(1)$ & 0.0029 & 0.0010 & 0.0014 \\
$\mathrm{~N}(1)$ & $-0.005(2)$ & -0.0018 & 0.0029 & 0.0003 \\
$\mathrm{~N}(2)$ & $-0.092(2)$ & -0.0794 & -0.0703 & -0.0746 \\
$\mathrm{C}(21)$ & $0.043(2)$ & 0.0422 & 0.0436 & 0.0404 \\
$\mathrm{C}(31)$ & $-0.107(2)$ & -0.1146 & -0.1146 & -0.1106 \\
$\mathrm{C}(51)$ & $0.037(2)$ & -0.0014 & 0.0190 & 0.0211 \\
$\mathrm{C}(61)$ & $0.008(2)$ & 0.0326 & 0.0211 & 0.0178 \\
$\mathrm{H}(1)$ & $-0.53(2)$ & -0.5859 & -0.5863 & -0.5833 \\
$\mathrm{H}(2)$ & $-0.24(2)$ & -0.3251 & -0.3063 & -0.3124 \\
$\mathrm{H}(3)$ & $0.25(2)$ & 0.3323 & 0.3540 & 0.3511 \\
$\mathrm{H}(4)$ & $0.28(2)$ & 0.3092 & 0.3131 & 0.2959 \\
\hline
\end{tabular}

${ }^{\mathrm{a}}$ Used in determining the least-squares mean plane. 
TABLE III. Selected bond lengths $(\AA)$ and angles $\left(^{\circ}\right)$ of DAD.

\begin{tabular}{lc}
\hline \hline $\mathrm{N}(1)-\mathrm{C}(1)$ & $1.424(2)$ \\
$\mathrm{N}(2)-\mathrm{C}(4)$ & $1.418(2)$ \\
$\mathrm{C}(1)-\mathrm{C}(2)$ & $1.403(2)$ \\
$\mathrm{C}(1)-\mathrm{C}(6)$ & $1.409(2)$ \\
$\mathrm{C}(2)-\mathrm{C}(3)$ & $1.405(2)$ \\
$\mathrm{C}(3)-\mathrm{C}(4)$ & $1.410(2)$ \\
$\mathrm{C}(4)-\mathrm{C}(5)$ & $1.405(2)$ \\
$\mathrm{C}(5)-\mathrm{C}(6)$ & $1.401(2)$ \\
$\mathrm{C}(2)-\mathrm{C}(21)$ & $1.513(2)$ \\
$\mathrm{C}(3)-\mathrm{C}(31)$ & $1.509(2)$ \\
$\mathrm{C}(5)-\mathrm{C}(51)$ & $1.509(2)$ \\
$\mathrm{C}(6)-\mathrm{C}(61)$ & $1.508(2)$ \\
$\mathrm{N}(1)-\mathrm{H}(1)$ & $0.91(2)$ \\
$\mathrm{N}(1)-\mathrm{H}(2)$ & $0.92(2)$ \\
$\mathrm{N}(2)-\mathrm{H}(3)$ & $0.95(2)$ \\
$\mathrm{N}(2)-\mathrm{H}(4)$ & $0.91(2)$ \\
$\mathrm{C}(2)-\mathrm{C}(1)-\mathrm{N}(1)$ & $121.1(1)$ \\
$\mathrm{C}(6)-\mathrm{C}(1)-\mathrm{N}(1)$ & $118.2(1)$ \\
$\mathrm{C}(3)-\mathrm{C}(4)-\mathrm{N}(2)$ & $119.1(1)$ \\
$\mathrm{C}(5)-\mathrm{C}(4)-\mathrm{N}(2)$ & $120.0(1)$ \\
$\mathrm{C}(1)-\mathrm{N}(1)-\mathrm{H}(1)$ & $111(1)$ \\
$\mathrm{C}(1)-\mathrm{N}(1)-\mathrm{H}(2)$ & $115(1)$ \\
$\mathrm{H}(1)-\mathrm{N}(1)-\mathrm{H}(2)$ & $110(1)$ \\
$\mathrm{C}(4)-\mathrm{N}(2)-\mathrm{H}(3)$ & $115(1)$ \\
$\mathrm{C}(4)-\mathrm{N}(2)-\mathrm{H}(4)$ & $111(1)$ \\
$\mathrm{H}(3)-\mathrm{N}(2)-\mathrm{H}(4)$ & $111(1)$ \\
$\mathrm{H}(22)-\mathrm{C}(21)-\mathrm{H}(23)$ & $105(2)$ \\
$\mathrm{H}(21)-\mathrm{C}(21)-\mathrm{H}(23)$ & $114(2)$ \\
$\mathrm{H}(31)-\mathrm{C}(31)-\mathrm{H}(33)$ & $105(2)$ \\
$\mathrm{C}(3)-\mathrm{C}(31)-\mathrm{H}(31)$ & $113(1)$ \\
$\mathrm{H}(51)-\mathrm{C}(51)-\mathrm{H}(52)$ & $94(2)$ \\
$\mathrm{C}(5)-\mathrm{C}(51)-\mathrm{H}(51)$ & $116(1)$ \\
$\mathrm{H}(61)-\mathrm{C}(61)-\mathrm{H}(63)$ & $102(2)$ \\
$\mathrm{C}(6)-\mathrm{C}(61)-\mathrm{H}(63)$ & $113(1)$ \\
\hline \hline & \\
\hline & \\
& \\
&
\end{tabular}

plane of benzene ring at a distance of 0.043(2) and 0.037(2) $\AA$, respectively, and the $\mathrm{C}(31)$ and $\mathrm{N}(2)$ atoms below this plane at a distance of $-0.107(2)$ and $-0.092(2) \AA$, respectively,. The similar values of the deviation from the planarity of these atoms were obtained in calculation using various functionals applied in the PW91 (DNP) method for the crystalline state of DAD. It should be noted that the deviation of the $\mathrm{C}(31)$ carbon atom and consequently of the methyl group attached to it is particularly large. Selected bond lengths and angles are given in Table III.

The hydrogen atoms of amino groups are displaced on opposite sites with respect to the benzene ring (Table II). When analyzing the bond angles of methyl groups (Table III) the disturbance of the $\mathrm{CH}$ bonds from the $s p^{3}$ hybridization is well visible. The bonding around the methyl group carbon atom is distorted from the ideal tetrahedral geometry. The values of the bond angles in this group are in the range between $94(2)^{\circ}$ and $116(1)^{\circ}$. Generally one can tell that in the solid state the DAD molecule completely loses symmetry of the free molecule.

From the point of view of packing and dynamics of methyl groups the conventional and unconventional hydrogen bonds are important. Their parameters are collected in Table IV, whereas the packing of the molecules in the lattice is illustrated in Fig. 2.
TABLE IV. Hydrogen bonds and short contacts of DAD.

\begin{tabular}{|c|c|c|c|c|}
\hline $\mathrm{D}-\mathrm{H} \cdots A$ & $\begin{array}{c}\mathrm{D} \cdots A \\
(\AA)\end{array}$ & $\begin{array}{l}\mathrm{D}-\mathrm{H} \\
(\AA)\end{array}$ & $\begin{array}{c}\mathrm{H} \cdots A \\
(\AA)\end{array}$ & $\underset{\left({ }^{\circ}\right)}{<\mathrm{D}-\mathrm{H} \cdots A}$ \\
\hline $\mathrm{N}(1)-\mathrm{H}(2) \cdots \mathrm{N}(2)^{\mathrm{a}}$ & $3.245(2)$ & $0.92(2)$ & $2.45(2)$ & $153(1)$ \\
\hline $\mathrm{N}(2)-\mathrm{H}(3) \cdots \mathrm{N}(1)^{\mathrm{b}}$ & $3.255(2)$ & $0.95(2)$ & $2.37(2)$ & $154(1)$ \\
\hline $\mathrm{C}(21)-\mathrm{H}(21) \cdots \mathrm{N}(1)$ & $2.890(2)$ & $0.96(2)$ & $2.57(2)$ & $99(1)$ \\
\hline $\mathrm{C}(31)-\mathrm{H}(32) \cdots \mathrm{N}(2)$ & $2.816(2)$ & $0.99(2)$ & $2.64(2)$ & $90(1)$ \\
\hline $\mathrm{C}(51)-\mathrm{H}(51) \cdots \mathrm{N}(2)$ & $2.867(2)$ & $0.96(3)$ & $2.51(2)$ & $102(2)$ \\
\hline $\mathrm{C}(61)-\mathrm{H}(61) \cdots \mathrm{N}(1)$ & $2.795(2)$ & $0.99(2)$ & $2.62(2)$ & $90(1)$ \\
\hline $\mathrm{C}(51)-\mathrm{H}(51) \cdots \mathrm{N}(1)^{\mathrm{b}}$ & $3.634(2)$ & $0.96(3)$ & $2.77(3)$ & $145(2)$ \\
\hline $\mathrm{C}(51)-\mathrm{H}(51) \cdots \mathrm{N}(1)^{\mathrm{c}}$ & $3.405(2)$ & $0.96(3)$ & $2.94(2)$ & $111(2)$ \\
\hline $\mathrm{C}(61)-\mathrm{H}(61) \cdots \mathrm{N}(2)^{\mathrm{d}}$ & $3.627(2)$ & $0.99(2)$ & $2.70(2)$ & $158(1)$ \\
\hline $\mathrm{C}(51)-\mathrm{H}(53) \cdots \mathrm{C}(2)^{\mathrm{e}}$ & $3.598(2)$ & $0.87(3)$ & $2.86(2)$ & $144(2)$ \\
\hline $\mathrm{C}(51)-\mathrm{H}(53) \cdots \mathrm{C}(3)^{\mathrm{e}}$ & $3.700(2)$ & $0.87(3)$ & $2.93(2)$ & $148(2)$ \\
\hline $\mathrm{C}(61)-\mathrm{H}(63) \cdots \mathrm{C}(3)^{\mathrm{f}}$ & $3.691(2)$ & $1.00(2)$ & $2.74(2)$ & $160(2)$ \\
\hline $\mathrm{C}(61)-\mathrm{H}(63) \cdots \mathrm{C}(4)^{\mathrm{f}}$ & $3.548(2)$ & $1.00(2)$ & $2.82(2)$ & $130(1)$ \\
\hline $\begin{array}{l}\mathrm{a}_{-}-x+1 / 2, y-1 / 2, z . \\
\mathrm{b}-x+1, y+1 / 2,-z+1 / \\
\mathrm{c}-x+1 / 2, y+1 / 2, z . \\
\mathrm{c}_{-}^{\mathrm{d}}-x+1, y-1 / 2,-z+1 / \\
\mathrm{e}^{\mathrm{e}}-1 / 2, y,-z+1 / 2 . \\
\mathrm{f}^{\mathrm{f}}-1 / 2, y,-z+1 / 2 .\end{array}$ & & & & \\
\hline
\end{tabular}

The fact that the conventional $\mathrm{N}-\mathrm{H} \cdots \mathrm{N}$ hydrogen bonds are rather weak (3.245(2), 3.255(2) $\mathrm{A}$ ) finds its expression in the IR spectra. There are quite marked differences between the two $\mathrm{NH}_{2}$ groups. All the $\mathrm{CH}_{3}$ groups form the intramolecular $\mathrm{C}-\mathrm{H} \cdots \mathrm{N}$ bonds $(2.57(2)-2.64(2) \AA)$ with the $\mathrm{H} \cdots \mathrm{N}$ distances close to the sum of the van der Waals radii [2.75 $\AA$ (Ref. 35]. Moreover the $\mathrm{N}-\mathrm{H} \cdots \mathrm{N}$ hydrogen bonds are strongly nonlinear.

Furthermore, the $\mathrm{N}(1)$ and $\mathrm{N}(2)$ atoms are also involved in the weak intermolecular $\mathrm{C}-\mathrm{H} \cdots \mathrm{N}$ hydrogen bonds. The $\mathrm{C}-\mathrm{H} \cdots \mathrm{C}_{\text {benzene }}$ contacts have some importance. The $\mathrm{H} \cdots \mathrm{C}_{\text {benzene }}$ distances are close to the sum of the van der Waals radii $(2.90 \AA)$. A detailed analysis of the intermolecular $\mathrm{H} \cdots \mathrm{H}$ contacts shows that they are markedly longer as compared to the sum of van der Waals radii $(2.40 \AA)$. The intermolecular $\mathrm{N}-\mathrm{H} \cdots \pi$ and $\mathrm{C}-\mathrm{H} \cdots \pi$ distances and angles are found as follows: for $\mathrm{N}(1)-\mathrm{H}(1) \cdots \pi(\mathrm{Cg})^{i}[\mathrm{H}(1) \cdots \pi$ $-2.96(2) \AA, \mathrm{N}(1) \cdots \pi-3.585(1) \AA$, and $\mathrm{N}(1)-\mathrm{H}(1) \cdots \pi$

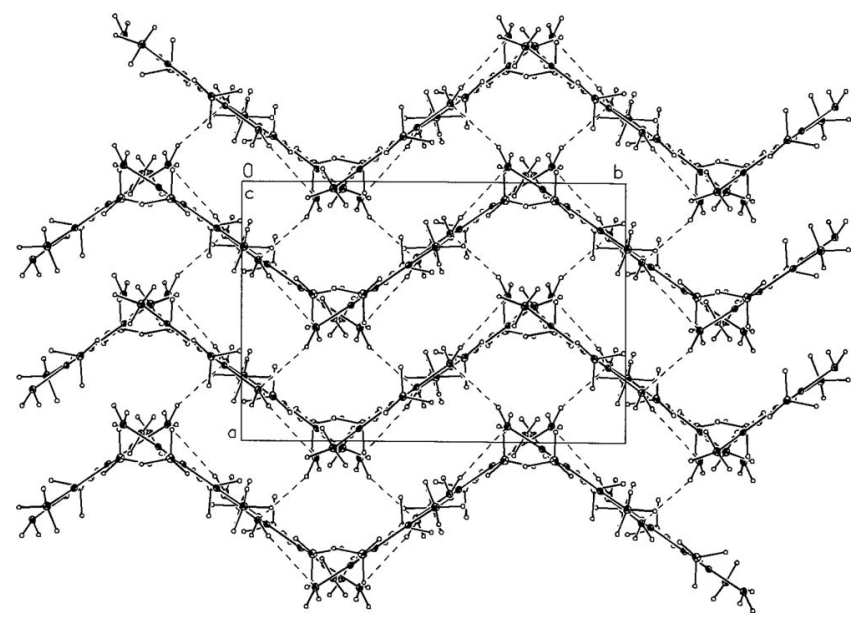

FIG. 2. Packing of molecules seen along the $c$-axis with dashed lines indicating $\mathrm{N}(1)-\mathrm{H}(2) \cdots \mathrm{N}(2)^{i}$ and $\mathrm{N}(2)-\mathrm{H}(3) \cdots \mathrm{N}(1)^{i i}$ hydrogen bonds. 


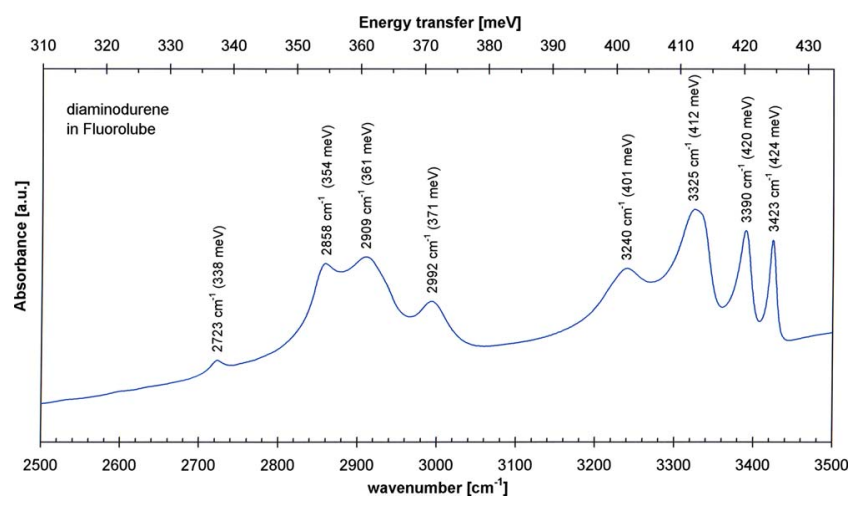

FIG. 3. (Color online) Infrared spectrum of DAD in the region of the $\nu\left(\mathrm{CH}_{3}\right)$ and $\nu\left(\mathrm{NH}_{2}\right)$ stretching vibrations.

$\left.-128(1)^{\circ}\right] \quad$ and $\quad \mathrm{C}(51)-\mathrm{H}(53) \cdots \pi(\mathrm{Cg})^{i i} \quad[\mathrm{H}(53) \cdots \pi$ $-2.96(2) \AA, \quad \mathrm{C}(51) \cdots \pi-3.524(2) \AA, \quad$ and $\left.\mathrm{C}(51)-\mathrm{H}(53) \cdots \pi-124(2)^{\circ}\right]$, where $\mathrm{Cg}^{i}(-1 / 2+x, y, 1 / 2$ $+z)$ and $\mathrm{Cg}^{i i}(1 / 2+x, y, 1 / 2-z)$ denote the centers of gravity of benzene rings.

Of some importance, from the point of view of packing and dynamics of particular atoms and groups, could be an analysis of the anisotropic displacement parameters. The $U_{\text {eq }}$ values for the $\mathrm{C}(21)$ and $\mathrm{C}(31)$ carbon atoms equal $0.0269(3) \AA^{2}$, while they are $0.0242(3)$ and $0.0235(3) \AA^{2}$ for the $C(51)$ and $C(61)$, respectively. This means that the two latter methyl groups are more rigidly built into the lattice.

\section{B. Infrared and Raman spectra}

The infrared spectrum of DAD recorded in Fluorolube suspension in the high frequency range is shown in Fig. 3. The analysis of the spectrum was based on the calculated frequencies by using GAUSSIAN 03 program 27 on the $B 3 L Y P / 6-31 G(d, p)$ level of theory. A scaling factor equal to 0.95 was applied. In the calculated $\nu\left(\mathrm{CH}_{3}\right)$ frequencies three (unresolved) quartets are predicted at 2987-3005, 2910, and 2854-2856 $\mathrm{cm}^{-1}$, i.e., there is a rather good agreement with experimental trio at 2992, 2909, and $2858 \mathrm{~cm}^{-1}$. The $\nu\left(\mathrm{NH}_{2}\right)$ vibrations of the free molecule are calculated at 3486

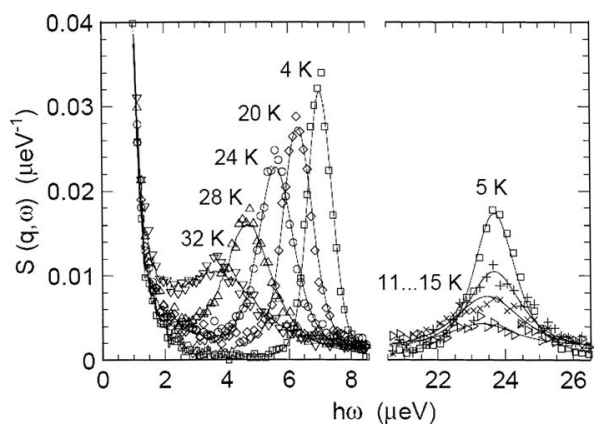

FIG. 4. Tunneling spectra of diaminodurene measured with the neutron backscattering spectrometers IN16 (left frame, energy range of $\pm 10 \mu \mathrm{eV}$, and concentrating on tunneling peak 1) and SPHERES (right frame, energy range of $\pm 31 \mu \mathrm{eV}$, and concentrating on low temperatures where tunneling peak 2 shows a rapid softening). Wavelength of $6.27 \AA$; averaged over all large-angle detectors $\left(Q=0.6-1.9 \AA^{-1}\right)$. Solid lines are fits.

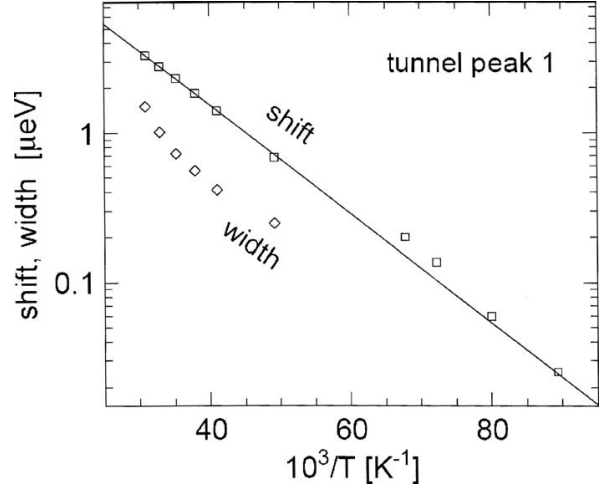

FIG. 5. Shift in and broadening of tunneling peak 1 as function of reciprocal temperature. The solid line shows Arrhenius behavior with an activation energy of $7.2 \mathrm{meV}$.

and $3395 \mathrm{~cm}^{-1}$, which almost exactly correspond to the situation in aniline, ${ }^{36} 3481$ and $3395 \mathrm{~cm}^{-1}$. In our case one observes four peaks $3423+3325$ and $3390+3240 \mathrm{~cm}^{-1}$ corresponding to $\nu_{\mathrm{as}}\left(\mathrm{NH}_{2}\right)$ and $\nu_{\mathrm{s}}\left(\mathrm{NH}_{2}\right)$ modes of two different $\mathrm{NH}_{2}$ groups. This result confirms the formation of different $\mathrm{N}-\mathrm{H} \cdots \mathrm{N}$ hydrogen bonds by the particular $\mathrm{NH}_{2}$ groups. The shift in $\nu\left(\mathrm{NH}_{2}\right)$ bands toward low frequencies is not very high, and the hydrogen bonds should be classified as rather weak. In the wavenumber region corresponding to the $\partial\left(\mathrm{NH}_{2}\right)$ vibrations, only one band at $1631 \mathrm{~cm}^{-1}$ (infrared) and at $1630 \mathrm{~cm}^{-1}$ (Raman) is observed.

\section{Neutrons: Rotational tunneling}

For backscattering, the widest energy range of $\pm 31 \mu \mathrm{eV}$ is available at SPHERES. On this spectrometer, two tunneling transitions of equal intensities but different widths are detected at 7.0 and $23.7 \mu \mathrm{eV}$ (Fig. 4). The outer peak broadens rapidly on heating from 4 to $15 \mathrm{~K}$. To study the evolution of the inner peak, which extends over a much wider temperature range, we used IN16 with a dynamic range of $\pm 10 \mu \mathrm{eV}$. Extending the energy range by using the TOFTOF spectrometer, no further transitions were observed up to the free rotor energy of $0.75 \mathrm{meV}$.

The ratio of elastic intensity to that on a single tunneling transition is 22. Equation (3) yields this value for momentum transfer of $Q=1.64 \AA^{-1}$, corresponding to a mean scattering angle $2 \Theta=110^{\circ}\left(j_{o}=0.1\right)$. To conclude, from the four inequivalent methyl groups identified by the x-ray structure determination, two tunnel splittings are resolved and two others are not resolved.

With increasing temperature, the outer tunneling peak broadens so rapidly that a quantitative analysis of the tem-

TABLE V. Parameters of Arrhenius functions characterizing the broadening of tunneling and quasielastic spectra of DAD.

\begin{tabular}{lcc}
\hline \hline Component & $\Gamma_{\mathrm{oi}}(\mathrm{meV})$ & $E_{\mathrm{ai}}(\mathrm{meV})$ \\
\hline Quasielastic 1 & 4.0 & 14 \\
Quasielastic 2 & 4.0 & 27 \\
Quasielastic 3 & 4.0 & 43 \\
Tunneling shift 1 & 0.044 & 7.2 \\
\hline \hline
\end{tabular}




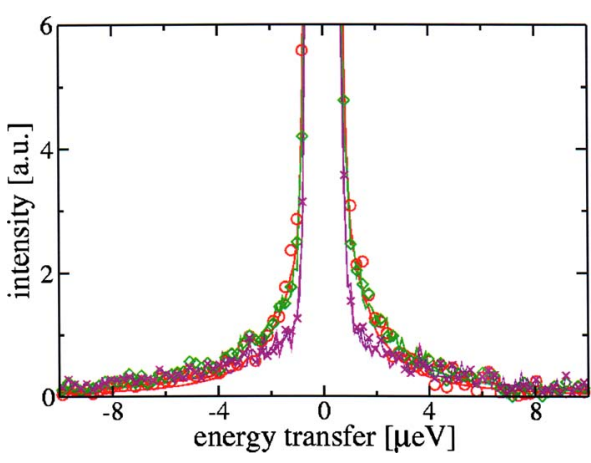

FIG. 6. (Color online) Quasielastic spectra of DAD. Sample temperatures were $50 \mathrm{~K}(\bigcirc), 60 \mathrm{~K}(\diamond)$, and $70 \mathrm{~K}(\times)$. Spectrometer: IN16 of ILL. $\lambda$ $=6.27 \AA . Q=1.6 \AA^{-1}$. The solid lines are fits.

perature dependence is not possible. The softening of the inner peak follows Arrhenius' dependence with an activation energy of $7.2 \mathrm{meV}$ (Fig. 5, Table V). The broadening of the inner peak is slightly curved in the Arrhenius plot. This result, however, is uncertain; for high temperatures, where the wing of the tunneling line overlaps with the wing of the elastic line, fit results are very sensitive to the way the instrumental resolution is modeled. While further studies are pending, at present no error bars can be given. In the low temperature-limit, the activation energy possibly coincides with the $7.2 \mathrm{meV}$ of the softening.

According to the perturbation theory of coupling a quantum rotor to phonon ${ }^{37}$ this energy should reflect the resonant coupling to phonons with the energy of the librational mode and, therefore, represent its librational energy $E_{01}$. These results will be discussed when we derive rotational potentials.

\section{Neutrons: Quasielastic spectra}

Several quasielastic spectra from IN16 are shown in Fig. 6 . The energy resolution of the backscattering instrument is too good for a reliable exploration of classical dynamics of weakly hindered methyl groups. At temperature, where the dynamical process is fully classical, most of the quasielastic intensity has already left the energy window of the instrument.

With its reduced but still high energy resolution quasielastic spectra were measured on the cold TOF instrument TOFTOF of the Technical University München at FRMII in

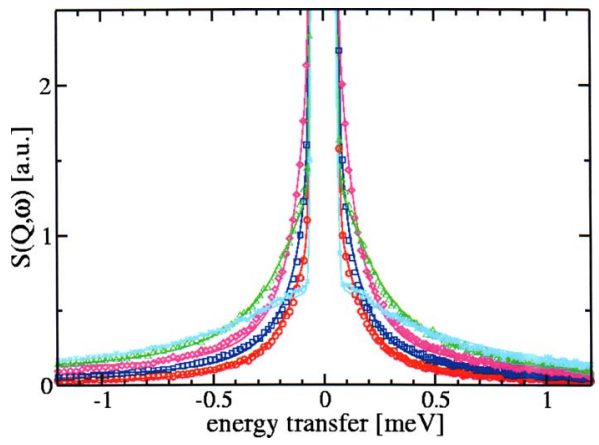

FIG. 7. (Color online) Quasielastic spectra of diaminodurene. Sample temperatures were $50 \mathrm{~K}(\bigcirc), 70 \mathrm{~K}(\square), 110 \mathrm{~K}(\diamond), 150 \mathrm{~K}(\triangle)$, and $200 \mathrm{~K}(\times)$. Spectrometer TOFTOF of TUM at FRMII. $\lambda=6.0 \AA . . Q=1.7 \AA^{-1}$. The solid lines are fits.

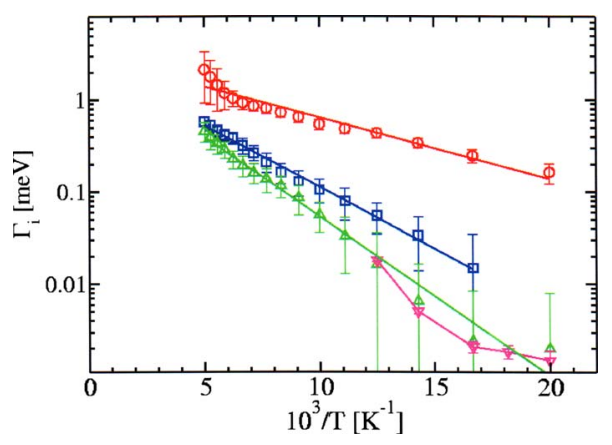

FIG. 8. (Color online) Arrhenius fits of the widths of the three quasielastic components in neutron spectra of TOFTOF and IN16.

the temperature range from 50 to $220 \mathrm{~K}$. The quasielastic intensity is a superposition of contribution from all inequivalent methyl groups. We model the scattering by a deltafunction and three Lorentzians of intensity ratio 1:1:1 and individual linewidths. The justification to omit the fourth Lorentzian is given by the strong potentials of the fourth methyl group (see below). The temperature evolution of the phonon spectrum is taken into account by a Debye spectrum. A subset of spectra out of the 17 scans is shown in Fig. 7.

Figure 8 shows the extracted linewidths as a function of the reciprocal temperature. The few points measured on IN16 fall nicely on the steepest line corresponding to the most strongly hindered group. The parameters obtained from an Arrhenius fit are shown in Table V, upper part. All obtained activation energies are rather low.

\section{E. Neutrons and mathematical modeling: Vibrational spectra}

The INS spectra obtained by using various neutron spectrometers are presented in Fig. 9. The broadest frequency region, up to $1200 \mathrm{~cm}^{-1}$, is obtained by using the IN1-BeF spectrometer. At frequencies below $300 \mathrm{~cm}^{-1}$ more detailed data are observed by using the IN4 $(\lambda=1.5 \AA)$ spectrometer.

The comparison of experimental frequencies obtained from INS as well as in infrared and Raman spectra and those calculated by using various DFT methods is presented in supplementary Table S1. ${ }^{38}$ Here we analyze in more details

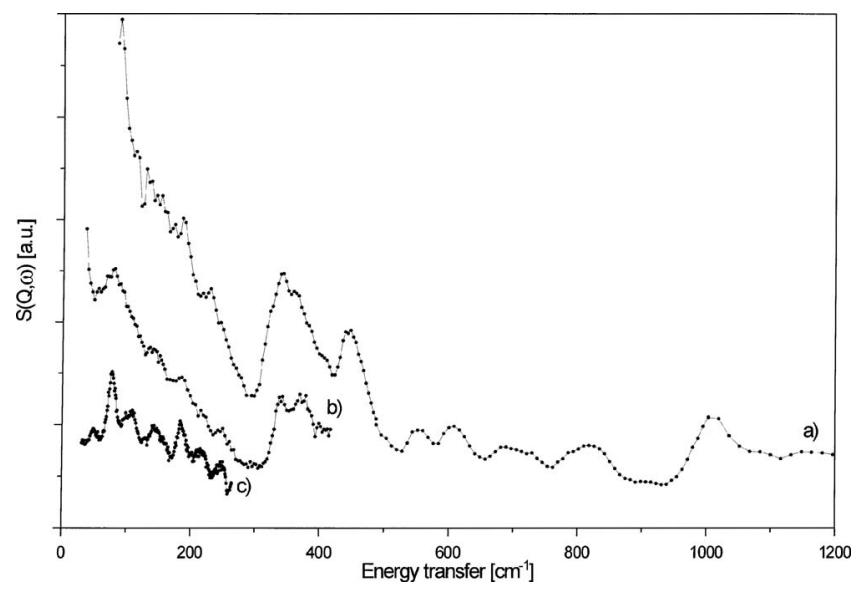

FIG. 9. INS vibrational spectra recorded by (a) IN1-BeF, (b) IN4 $(\lambda$ $=1 \AA)$, and (c) IN4 ( $\lambda=1.5 \AA)$ spectrometers. 
TABLE VI. Calculated and experimental (INS) energies (meV) for transitions in DAD in the range of the $\mathrm{CH}_{3 \text { tors }}$ modes.

\begin{tabular}{|c|c|c|c|c|c|}
\hline \multirow[b]{2}{*}{ Assignment } & \multicolumn{4}{|c|}{ Calculated } & \multirow{2}{*}{$\frac{\text { Experimental }}{\text { INS }}$} \\
\hline & B3LYP/6-31G** & BLYP(DNP) & PBE(DNP) & PW91(DNP) & \\
\hline Ring tors $+\mathrm{CH}_{3 \text { tors }}$ & 10.0 & 8.7 & 8.7 & 8.7 & 9.5 \\
\hline Ring tors $+\mathrm{CH}_{3 \text { tors }}$ & 11.2 & 10.5 & 10.5 & 10.5 & 10.9 \\
\hline $\mathrm{CH}_{3 \text { tors }}+$ ring tors & 13.0 & 13.1 & 13.4 & 13.6 & 13.5 \\
\hline $\mathrm{CH}_{3 \text { tors }}+$ ring tors & 15.3 & 17.7 & 16.9 & 16.9 & 17.9 \\
\hline $\mathrm{CH}_{3 \text { tors }}$ & 17.0 & 19.2 & 20.2 & 18.8 & 19.3 \\
\hline $\mathrm{CH}_{3 \text { tors }}$ & 19.3 & 25.3 & 24.3 & 22.7 & 22.9 \\
\hline
\end{tabular}

the data related to the lowest frequencies corresponding to $\mathrm{CH}_{3}$ torsional vibrations, as presented in Table VI. It is remarkable that the four lowest modes are ring torsions mixed with methyl librations. Two further modes correspond to the neat $\mathrm{CH}_{3}$ torsional vibrations. The frequencies of these and the other librational/torsional modes of $\mathrm{CH}_{3}$ groups are in the range expected from the tunneling splittings and QENS spectra. Details are discussed in the following section.

We would like to direct attention to the following details. The calculated frequencies for free molecules are markedly lower than the experimental ones. This refers particularly to the neat $\mathrm{CH}_{3 \text { tors }}$ modes. Quite good consistency with experiment is obtained by using methods elaborated for the crystalline state. The best consistency with experiment was achieved for the PW91 (DNP) method. The differences between results obtained for free molecule and for the crystalline state can be discussed in terms of unconventional hydrogen bonds with participation of $\mathrm{CH}_{3}$ groups. The largest differences between internal frequencies calculated for the free molecules and those measured and calculated for the crystalline state are found for bending modes of $\mathrm{NH}_{2}$ groups. The calculated values for free molecule are dramatically lower. This seems to be understandable in the light of conventional hydrogen bonds. As it is commonly known this type of interactions leads (in contrast to stretching vibrations) to a marked increase in frequencies. Finally one has to realize that the modes important for our analysis, i.e., the $\mathrm{CH}_{3}$ torsional ones, are practically unobservable in infrared and Raman spectra.

\section{F. Rotational potentials}

An appropriate potential has to reproduce the three quantities: tunnel splitting, librational energy, and barrier height. Such potentials have been investigated. If this search is done systematically for all groups by combining the largest tunnel splitting with the lowest librational mode-assigned to a tunneling mode by its broadening with temperature- $\mathrm{a}$ tunnel transition around $100 \mu \mathrm{eV}$ should be found. A transition at this energy was not observed however. Our hypothesis is that the very low lying ring torsion (Table VI) influences the temperature dependence of the tunnel bands and couples strongly to methyl torsions. Therefore possibly the ring torsion may appear as activation energy of the broadening of a tunnel transition. Furthermore the coupling between ring torsion and methyl libration leads to dispersion. Peaks in the density of states may not be viewed as single particle torsional modes. (This hypothesis shall be tested in a forthcoming experiment by modifying the energy of these modes using $\left(\mathrm{ND}_{2}\right)_{2}$-DAD.) The potentials shown in Table VII therefore reproduce just the tunneling splittings $\hbar \omega_{t}$ and the activation energies. The calculated librational energies (Table VII) are higher than those derived from the temperature evolution of the tunneling bands as shown in Table V.

For the most strongly hindered methyl group the librational energy of $22 \mathrm{meV}$ is well above (translational) phonon energies and thus decoupled from the phonon spectrum. From $E_{o 1}=22 \mathrm{meV}$ a leading $\cos (3 \varphi)$ term of the potential can be determined. The activation energy for this most strongly hindered methyl group, likely the one with the lowest values of anisotropic displacement parameters, C(61), is so high that the calculated quasielastic linewidth is even at the highest temperature used in our experiment clearly below one-tenth of the resolution of the TOFTOF spectrometer. Therefore only three quasielastic components of equal intensities were found.

\section{CONCLUSIONS}

DAD, as an object of studies on the dynamics of methyl groups, seems to be interesting from various points of view. It contains, in addition to four methyl groups, two amino

TABLE VII. Rotational potentials of DAD adjusted for tunnel splittings $\hbar \omega_{t}$ (if available) and activation energies $E_{a}$. Values in brackets are calculated.

\begin{tabular}{cccccc}
\hline \hline & & & & \multicolumn{2}{c}{ Potential $(\mathrm{meV})$} \\
\cline { 5 - 6 } Label & $\hbar \omega_{t}(\mu \mathrm{eV})$ & $E_{o 1}(\mathrm{meV})$ & $E_{a}(\mathrm{meV})$ & $V_{3}$ & $V_{6}$ \\
\hline 1 & 23.5 & $(10.8)$ & 14 & 20.8 & 5 \\
2 & 6.85 & $(12.2)$ & 27 & 33.6 & -0.3 \\
3 & $(0.99)$ & $(15.7)$ & 43 & 51.3 & $\ldots$ \\
4 & $(0.02)$ & 22 & & 84 & $\cdots$ \\
\hline \hline
\end{tabular}


groups, which cause relatively strong electrodonor properties, thus belonging to active components of the CT complexes. This was the reason of its selection in studies on the influence of the CT degree on the rotational potential of methyl groups.

So far the structure of DAD was not known so that the first step in our investigations was an x-ray diffraction study. The packing of molecules in the crystalline lattice of DAD leads to the complete loss of symmetry of the molecule, and as a consequence we are dealing with four different methyl groups and two different amino groups. The hydrogen bonds with the latter groups play an essential role in the packing so that the lone electron pairs at nitrogen atoms are markedly blocked. However, the analysis shows that in the lattice both intra- and intermolecular $\mathrm{C}-\mathrm{H} \cdots \mathrm{N}$ bonds exist between $\mathrm{CH}_{3}$ and $\mathrm{NH}_{2}$ groups. As the analysis has shown, the $\mathrm{CH}_{3}$ groups with $\mathrm{C}(31)$ carbon atoms are seriously distorted in the solid state that is spectacularly reflected by the displacement of these groups out of the plane of the benzene ring plane. With increasing distance to nearest neighbors the rotational potential of this methyl group weakens. The group with the distinctly strongest increase in the rotational barrier is that including $\mathrm{C}(61)$. This group also has a distinctly stronger rotational barrier than the other ones and its tunneling splitting cannot be resolved. The torsional vibrations manifested in INS spectra are to a large extent coupled with the ring vibrations and located in the lowest frequency region between 80 and $180 \mathrm{~cm}^{-1}$. The low rotational barriers are clearly reflected in the large tunneling splittings.

In DAD a coupling between the ring torsions and methyl librations is the very interesting point. The effect of the coupling on the temperature dependence of the tunneling bands shall be investigated in a forthcoming experiment.

In summarizing one gets a consistent picture of the dynamics, especially of methyl groups, in the simple molecular crystal as DAD, which appeared, however, complicated due to the packing effects of the soft molecules in the crystalline lattice.

\section{ACKNOWLEDGMENTS}

The calculations were performed on computers of the Wroclaw Centre for Networking and Supercomputing, under Calculating Grant No. 2006/5, and in Interdisciplinary Centre for Mathematical and Computational Modeling, Warsaw University, under Calculating Grant No. G30-15.

MATERIALS STUDIO package was used under POLAND COUNTRY-WIDE LICENSE.

This work was supported by the Polish Ministry of Science and Higher Education (Project Register No. N N204 $249734)$.

${ }^{1}$ P. C. H. Mitchell, S. F. Parker, A. J. Ramirez-Cuesta, and J. Tomkinson, Vibrational Spectroscopy with Neutrons (World Scientific, London, 2005).

${ }^{2}$ A. Pawlukojć and L. Sobczyk, Trends Appl. Spect. 5, 117 (2004).
${ }^{3}$ S. F. Parker, in Handbook of Vibrational Spectroscopy, edited by J. Chalmers and P. R. Griffiths (Wiley, New York, 2002), Vol. 1, pp. 838852.

${ }^{4}$ T. Springer, Quasielastic Neutron Scattering for the Investigation of Diffusive Motions in Solids and Liquids, Springer Tracts in Modern Physics Vol. 64 (Springer, Berlin, 1972).

${ }^{5}$ M. Bee, Quasielastic Neutron Scattering (Adam Hilger, Bristol, 1988).

${ }^{6}$ M. Prager and A. Heidemann, Chem. Rev. (Washington, D.C.) 97, 2933 (1997).

${ }^{7}$ W. Press, Single Particle Rotations in Molecular Crystals, Springer Tracts in Modern Physics Vol. 81 (Springer, Berlin, 1981).

${ }^{8}$ M. R. Johnson and G. J. Kearley, Annu. Rev. Phys. Chem. 51, 297 (2000).

${ }^{9}$ M. R. Johnson, M. Prager, H. Grimm, M. A. Neumann, G. J. Kearley, and C. C. Wilson, Chem. Phys. 244, 49 (1999).

${ }^{10}$ M. Plazanet, M. R. Johnson, J. D. Gale, T. Yildirim, G. J. Kearley, M. T. Fernández-Díaz, D. Sánchez-Portal, E. Artacho, J. M. Soler, P. Ordejón, A. Garcia, and H. P. Trommsdorff, Chem. Phys. 261, 189 (2000).

${ }^{11}$ G. Bator, L. Sobczyk, A. Pawlukojć, J. Nowicka-Scheibe, E. Grech, J. Krawczyk, M. Nowina Konopka, I. Natkaniec, I. V. Kalinin, and O. Steinsvoll, Phase Transitions 80, 489 (2007).

${ }^{12}$ A. Pawlukojć, I. Natkaniec, G. Bator, L. Sobczyk, E. Grech, and J. Nowicka-Scheibe, Spectrochim. Acta, Part A 63, 766 (2006).

${ }^{13}$ W. Sawka-Dobrowolska, G. Bator, B. Czarnik-Matusewicz, L. Sobczyk, A. Pawlukojć, J. Nowicka-Scheibe, E. Grech, and H. Rundlof, Chem. Phys. 327, 237 (2006).

${ }^{14}$ M. Prager, A. Pawlukojć, L. Sobczyk, E. Grech, and H. Grimm, J. Phys.: Condens. Matter 17, 5725 (2005).

${ }^{15}$ M. Prager, A. Wischnewski, G. Bator, E. Grech, A. Pawlukojć, and L. Sobczyk, Chem. Phys. 334, 148 (2007).

${ }^{16}$ A. M. M. Rawashdeh, C. Sotirion-Leventis, X. Gao, and N. Leventis, Chem. Commun. (Cambridge) 2001, 1742.

${ }^{17}$ L. Michaelis, M. P. Schubert, and S. Granick, J. Am. Chem. Soc. 61, 1981 (1939)

${ }^{18}$ M. Bose and M. M. Labes, J. Am. Chem. Soc. 83, 4505 (1961).

${ }^{19}$ R. A. Bullen, T. C. Arnot, J. B. Lakeman, and F. C. Walsh, Biosens. Bioelectron. 21, 2015 (2006).

${ }^{20}$ V. D. Samuilov, E. M. Lagunova, E. V. Dzyubinskaya, D. S. Izyumov, D. B. Kiselevsky, and Ya. V. Makarova, Biochemistry (Mosc.) 67, 627 (2002).

${ }^{21}$ G. M. Sheldrick, SHELXS-97 Program for Solution of Crystal Structure, University of Göttingen, 1997.

${ }^{22}$ G. M. Sheldrick, SHELXL-97 Program for Crystal Structure Refinement, University of Göttingen, 1997.

${ }^{23} \mathrm{http}: / / \mathrm{www} . i 11 . e u / t o f / i n s t r u m e n t s$

${ }^{24} \mathrm{http} / / / \mathrm{www} . j \mathrm{cns}$.info/jens_spheres

${ }^{25}$ J. Wuttke, G. J. Schneider, and L. C. Pardo, Z. Phys. Chem. (submitted).

${ }^{26}$ T. Unruh, J. Neuhaus, and W. Petry, Nucl. Instrum. Methods Phys. Res. A 380, 1414 (2002).

${ }^{27}$ M. J. Frisch, G. W. Trucks, H. B. Schlegel, G. E. Scuseria, M. A. Robb, J. R. Cheesman, J. A. Montgomery, T. Vreven, K. N. Kudin, J. C. Burant, J. M. Millan, S. S. Iyenger, J. Tomasi, V. Barone, B. Mennucci, M. Cossi, G. Scalmani, N. Rega, G. A. Peterson, H. Nakatsuji, M. Hada, M. Ehara, K. Toyota, R. Fukada, J. Hasegawa, M. Ishida, T. Nakajima, Y. Honda, O. Kitao, H. Nakai, M. Klene, X. Li, J. E. Knox, H. P. Hratchian, J. B. Cross, V. Bakken, C. Adamo, J. Jaramillo, R. Gomperts, R. E. Stratmann, O. Yazyev, A. J. Austin, R. Cammi, C. Pomelli, J. Ochterski, P. Y. Ayala, O. Cui, K. Morokuma, G. A. Voth, P. Salvador, J. J. Dennenberg, V. G. Zakrzewski, S. Dapprich, A. D. Daniel, M. C. Stain, D. K. Malick, A. D. Rabuck, K. Raghacachari, J. B. Foresman, J. V. Ortiz, O. Farkas, Q. Cui, A. G. Babul, S. Clifford, J. Cisowski, B. B. Stefanov, G. Liu, A. Liashenko, P. Piskorz, I. Komaromi, R. J. Martin, D. J. Fox, T. Keith, M. A. Al.-Laham, C. Y. Peng, A. Nanayakkara, M. Hallacombe, P. M. W. Gill, B. G. Johnson, W. Chen, M. W. Wong, C. Gonzales, and J. A. Pople, GAUSSIAN 03, Revision 6.0, Gaussian, Inc. Wallingford, CT, 2004.

${ }^{28}$ B. Delley, J. Chem. Phys. 92, 508 (1990).

${ }^{29}$ B. Delley, J. Chem. Phys. 113, 7756 (2000).

${ }^{30} \mathrm{http}: / / \mathrm{www}$.accelrys.com

${ }^{31}$ A. D. Becke, J. Chem. Phys. 88, 2547 (1988).

${ }^{32}$ C. Lee, W. Yang, and R. G. Parr, Phys. Rev. B 37, 786 (1988).

${ }^{33}$ J. P. Perdew, K. Burke, and M. Ernzerhof, Phys. Rev. Lett. 77, 3865 (1996).

${ }^{34}$ J. P. Perdew and Y. Wang, Phys. Rev. B 45, 13244 (1992).

${ }^{35}$ A. Bondi, J. Phys. Chem. 68, 441 (1964). 
${ }^{36}$ L. J. Bellamy, The Infrared Spectra of Complex Molecules (Wiley, New York, 1954).

${ }^{37}$ A. C. Hewson, J. Phys. C 15, 3841, (1982); 15, 3855, (1982).

${ }^{38}$ See EPAPS Document No. E-JCPSA6-130-023918 for Table S1. The comparison of experimental frequencies obtained from INS, infrared, and Raman spectra and those calculated by using various DFT methods. For more information on EPAPS, see http://www.aip.org/ pubservs/epaps.html. 